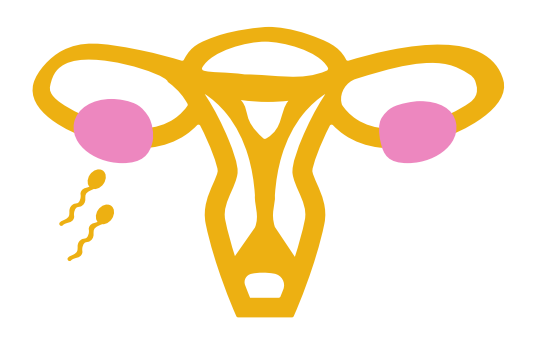

\title{
PRESERVAÇÃO DA FERTILIDADE EM MULHERES COM CÂNCER DE OVÁRIO
}

\author{
FERTILITY PRESERVATION IN WOMEN WITH OVARIAN CANCER
}

\begin{abstract}
Luana Maria Galdino da Silva Vilar
Enfermeira pela Universidade Potiguar - UnP, pós-graduada em Oncologia e Gestão Hospitalar e de Serviços de Saúde. Email: luanavilar_jc@yahoo.com.br

Danielle Karla de Araújo Duarte

Enfermeira pela Universidade Potiguar - UnP, pós-graduada em Gestão Hospitalar e de Serviços de Saúde.

Email: daniellekarla7@yahoo.com.br

Donátila Cristina Lima Lopes

Enfermeira pela Universidade Potiguar - UnP, pós-graduada em Gestão Hospitalar e de Serviços de Saúde.

Email: donatila.lima@gmail.com

Joana Gabryella Maia da Silva

Enfermeira pela Universidade Potiguar - UnP, pós-graduada em Gestão Hospitalar e de Serviços de Saúde.

Email: jjoanagaby@gmail.com

Robson Edney Mariano N. e Silva

Enfermeiro, pós-graduado em Enfermagem e docente da Universidade Potiguar - UnP
\end{abstract}

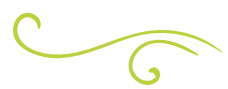

\section{RESUMO}

Este trabalho apresenta um apanhado teórico sobre as técnicas de preservação da fertilidade em mulheres acometidas por câncer ovariano, associado a técnicas de reprodução assistida. Há uma estimativa de que as mulheres acometidas por essa doença ainda estariam em período reprodutivo. Esta pesquisa utilizou como metodologia uma revisão integrativa, realizada por meio de levantamento bibliográfico feito no acervo da Biblioteca Virtual de Saúde (BVS). Este estudo pretende identificar na literatura nacional o quantitativo de publicações sobre a atuação do enfermeiro diante do diagnóstico de câncer ovariano e conhecer a fonte de informação que esses pacientes portadores de câncer ovariano tiveram acerca das técnicas de preservação.
Os artigos encontrados tratam do diagnóstico precoce da doença, situações em que a paciente tem a maior possibilidade de garantir a fertilidade. $O$ uso das técnicas de criopreservação de embriões, de oócitos, de tecido ovariano, supressão da função ovariana, e de transposição ovariana foram as técnicas encontradas para promover a preservação da fertilidade. $O$ trabalho desenvolvido mostra que devemos refletir sobre a atuação dos profissionais de enfermagem frente aos desejos dos pacientes oncológicos. Este trabalho buscou, no entanto, levantar informativos e estudos sobre a atuação do enfermeiro diante do diagnóstico de câncer e acerca das técnicas de preservação da fertilidade, mas houve certa dificuldade, devido à ausência de publicação relacionada ao tema. A atuação 
do enfermeiro junto a equipe multiprofissional e interdisciplinar precisa considerar o cuidado, priorizando os recursos, e com isso integrar os seus conhecimentos na assistência no ensino e em pesquisas em oncologia.

PALAVRAS-CHAVE:

Oncologia.

Neoplasias Ovarianas. Técnicas de preservação da fertilidade. Reprodução assistida.

\section{ABSTRACT}

This work presents a theoretical overview of the techniques of preservation of fertility in women affected by ovarian cancer, associated with assisted reproduction techniques. There is an estimate that the women affected by this disease would still be in the reproductive period. This research used as a methodology an integrative review, carried out by means of a bibliographical survey done in the collection of the Virtual Health Library. This study intends to identify in the national literature the quantitative of publications about the nurse's role in the diagnosis of ovarian cancer and to know the source of information that these patients with ovarian cancer had about preservation techniques. The articles found deal with the early diagnosis of the disease, situations in which the patient has the greatest possibility of guaranteeing fertility. The use of embryo cryopreservation, oocyte, ovarian tissue, suppression of ovarian function, and ovarian transposition techniques were the techniques used to promote fertility preservation. The work developed shows that we must reflect on the performance of nursing professionals in front of the wishes of cancer patients. This work, however, sought to collect information and studies about the nurse's role in the diagnosis of cancer and on the techniques of preservation of fertility, but there was some difficulty due to the absence of publication related to the topic. The nurse's work with the multidisciplinary and interdisciplinary team must consider care, prioritizing the resources, and with that integrate their knowledge in the assistance in teaching and research in oncology.

KEYWORDS: Oncology. Ovarian neoplasms. Preservation techniques fertility. Assisted reproduction.

\section{INTRODUÇÃO}

Este trabalho apresentará um apanhado teórico sobre as técnicas de preservação da fertilidade em mulheres acometidas por câncer ovariano, associado a técnicas de reprodução assistida. $\bigcirc$ objetivo desse estudo é identificar na literatura nacional o quantitativo de publicações sobre atuação dos enfermeiros diante do diagnóstico de câncer ovariano e conhecer o método mais utilizado para a preservação da fertilidade.

Diante disso, é importante inferir que câncer é o nome dado a um conjunto de doenças que apresentam alterações na estrutura do DNA das células, causando seu crescimento desordenado, com capacidade para formar novos vasos sanguíneos. Estes, por sua vez, nutrirão as células e manterão suas atividades descontroladas, fazendo com que invadam os tecidos e órgãos. Assim, diante do elevado número de óbitos por câncer, aumentaram as preocupações de muitos estudiosos no Brasil e no mundo, bem como o desejo pela descoberta do tratamento dessa patologia (INCA, 2018).

A estimativa para biênio 2018-2019 é de 600 mil novos casos de câncer no Brasil, sendo que praticamente metade seriam em mulheres, das quais 8 a 10\% teriam menos de 40 anos (INCA, 2018). Taxas brutas de incidência a cada 100 mil mulheres no Brasil mostra um ranking epidemiológico em que os cânceres de mama $(29,5 \%)$, intestino $(9,4 \%)$, colo do útero $(8,1 \%)$, pulmão $(6,2 \%)$ e tireoide $(4,0 \%)$ figurarão entre os principais, e o câncer de ovário, apesar de não estar entre esses, apresenta percentual de 3,6\% (INCA, 2018).

Pouco frequente, o câncer de ovário é o tumor ginecológico mais difícil de ser diagnosticado e o de menor chance de cura. Cerca de 3/4 dos cânceres desse órgão 
apresentam-se em estágio avançado no momento do diagnóstico. A maioria dos tumores de ovário são carcinomas epiteliais, canceres que se iniciam nas células da superfície do órgão - o mais comum - ou tumores malignos de células germinativas. Este tipo de célula dá origem tanto aos ovócitos, chamados erroneamente de óvulos, quanto aos espermatozoides (INCA, 2018).

Esse elevado número de casos atinge uma quantidade significativa de mulheres, aquelas que se encontram em uma faixa etária abaixo de 44 anos, ou seja, em período reprodutivo ou com um provável desejo futuro de engravidar (FERREIRA; SOARES; MOTTA, 2011). Esse panorama levou os especialistas, preocupados com a situação, a buscarem métodos que garantissem a preservação da fertilidade (CASTELLOTTI; CAMBIAGHI, 2008).

Nesse contexto, para as mulheres que têm o diagnóstico precoce da doença, e não têm problema de fertilidade prévia, as chances de garantir a fertilidade antes do início do tratamento com radioterapia e quimioterapia aumentam. Esses medicamentos aceleram a perda da capacidade reprodutiva, que é afetada gradativamente a partir da primeira menstruação da mulher, quando, num processo natural, a quantidade de óvulos começa a diminuir (HARTT, 2014).

As terapias utilizadas para o tratamento das doenças oncológicas envolvem medicamentos citotóxicos com a alto poder de lesão de celular germinativas, especialmente óvulos, a quimioterapia e a radioterapia podem levar a perda precoce da função ovariana, principalmente por um aumento da taxa de atresia folicular (FERREIRA; SOARES; MOTTA, 2011).

Também são apresentadas outras complicações a essas pacientes, decorrentes dos tipos das drogas utilizadas, bem como da irradiação, da duração do tratamento, da via de administração e da idade, podendo apresentar prejuízo à vascularização e ao desenvolvimento uterino no processo de nidação (ROSA E SILVA, 2006). Ou seja, a técnica pode causar o aborto, a perda gestacional de segundo trimestre, parto pré-termo e baixo peso ao nascimento devido a essas questões (CASTELLOTTI; CAMBIAGHI, 2008).

Contudo, é possível que a radioterapia, quando realizada em regiões pélvicas, desenvolva a infertilidade, bem como o câncer de colo de útero, ovário, endométrio, entre outros. Pode, inclusive, danificar ou até destruir os ovários, ou ainda prejudicar a vascularização e o desenvolvimento uterino, de acordo com a idade da paciente no momento da irradiação, do tamanho e localização do tumor, e da intensidade da irradiação para a cura (CASTELLOTTI; CAMBIAGHI, 2008).

Nesse sentido, as complicações para as pacientes em tratamento radioterápico são diversas. Entre essas, destacamos: interrupção da produção de hormônios e início da menopausa; perda da função ovariana, impedindo assim a gestação com seus próprios óvulos; aumento da incidência do elevado número de aborto; perda gestacional do segundo trimestre; parto pré-termo; e baixo peso ao nascimento (ROSA E SILVA, 2006).

No caso do tratamento quimioterápico, deve-se levar em consideração alguns aspectos, tais como: a idade da paciente, a doença, se foi feito ou não tratamento prévio para a infertilidade, a via de administração da medicação e a dose da droga necessária para a cura da doença. Como consequência da quimioterapia, tem-se a destruição ovariana, atingindo as células germinativas, que dão origem aos óvulos e espermatozoides. Além disso, há consequências tardias para as pacientes, as disfunções sexuais, como: insatisfações sexuais, dispareunia de penetração e diminuição da libido (CASTELLOTTI; CAMBIAGHI, 2008).

Embora a radioterapia e a quimioterapia se apresentarem como possibilidades no tratamento desses tipos de doença, há 
uma outra opção no combate a essas patologias: as cirurgias castradoras. Tais cirurgias são, geralmente, as melhores opções para a cura da mulher, em razão do índice de cura aumentar em utilizando esse tipo de tratamento. Entretanto, esse tipo de cirurgia traz prejuízo para a saúde reprodutiva, pois define para sempre o futuro infértil dessas pacientes (CASTELLOTTI; CAMBIAGHI, 2008).

Nesse contexto, após o tratamento, seja ele radioterapia, quimioterapia ou cirurgia castradora, muitos pacientes erradicam a doença. No entanto, a erradicação poderá ter como consequência a infertilidade da paciente, pois esses tratamentos afetam temporariamente ou definitivamente a fertilidade. Assim, a partir dessa problematização, ginecologistas aprimoraram técnicas de preservação da fertilidade e de fertilização, a fim de garantir a essas mulheres a constituição de suas famílias. Para que isso ocorra, é importante que profissionais da saúde, principalmente médicos e equipe de enfermagem, tenham o conhecimento das técnicas existentes, possibilitando, assim, a orientação, discussão, o planejamento e a intervenção adequada para que o objetivo e desejo dessas pacientes sejam alcançados (FERREIRA; SOARES; MOTTA, 2011).

Essa expectativa de constituir uma família após o tratamento torna-se eficaz quando ocorre o diagnóstico precoce da doença, e, antes do início da radioterapia e quimioterapia, utilizam-se as técnicas de preservação da fertilidade (LUNARDI; FERREIRA; BERNUCl, 2013). Essas técnicas, que envolvem ambos os sexos, são: congelamento de embriões, congelamento de tecido ovariano, congelamento de óvulos e transposição dos ovários, associados às técnicas de fertilização assistida, congelamento do sêmen e congelamento de tecido testicular, levando assim o aumento da expectativa de fertilidade após o término do tratamento oncológico (HARTT, 2014). Nessas circunstâncias, a pesquisa pretende responder os seguintes questionamentos: Existem orientações sobre a preservação da fertilidade por parte dos profissionais de saúde? O enfermeiro orienta sobre as técnicas de fertilização?

Diante disso, este estudo torna-se relevante pelo fornecimento de um apanhado teórico acerca das descobertas de preservação da fertilidade associados às técnicas de fertilização assistida. Dessa forma, favorece o entendimento do profissional de saúde, podendo esclarecer e melhorar o planejamento familiar e a própria saúde da mulher, assim como da população acerca dos métodos.

A motivação para este estudo se deve ao aumento no índice de casos oncológicos no aparelho reprodutor feminino, além da observação de que as mulheres acometidas com câncer ovariano representam um quantitativo significativo, quando se avalia valores populacionais e outras doenças consideradas não transmissíveis. Ainda nesse contexto, foi vivenciado pelas autoras a fragilidade nas informações sobre a preservação da fertilidade em pacientes submetidos ao tratamento oncológico, durante o estágio supervisionado de conclusão do curso.

Nesse contexto, foram estabelecidos para este estudo dois objetivos. Quais sejam: identificar, na literatura nacional, o quantitativo de publicações sobre a atuação do enfermeiro diante do diagnóstico de câncer ovariano e conhecer a fonte de informação que esses pacientes portadores de câncer ovariano tiveram acerca das técnicas de preservação.

\section{METODOLOGIA}

O estudo trata de uma revisão integrativa, a qual é um método de pesquisa utilizado desde 1980, no âmbito da Prática Baseada em Evidências (PBE). Essa metodologia envolve a sistematização e publicação dos resultados de uma pesquisa bibliográfica em saúde para que possam ser úteis na assistência à saúde, acentuando a importância da pesquisa acadêmica na prática clínica (MENDES; SILVEIRA; GALVAO, 2008). 
A revisão integrativa inclui a análise de pesquisas relevantes que dão suporte para a tomada de decisão e a melhoria da prática clínica, possibilitando a síntese do estado do conhecimento de um determinado assunto, além de apontar lacunas do conhecimento que precisam ser preenchidas com a realização de novos estudos (MENDES; SILVEIRA; GALVAO, 2008). Assim, esta pesquisa segue os meios determinados por tal metodologia com relação à organização dos dados.

A fim de responder o objetivo desta pesquisa, optou-se pelas seguintes questões norteadoras: Existem orientações sobre a preservação da fertilidade por parte dos profissionais de saúde? O enfermeiro orienta sobre as técnicas de fertilização e, quais as técnicas de fertilização mais utilizadas?

A busca pelo aporte teórico deste estudo foi realizada no período de julho a agosto de 2018, sendo levantados vários artigos, porém, apenas quatro foram utilizados, por atenderem aos objetivos propostos. Utilizou-se a Biblioteca Virtual de Saúde (BVS) com as bases de dados da Literatura Latino Americana e do Caribe em Ciências de Saúde (LILACS), Scientific Electronic Library (Scielo), bem como fontes do Ministério da Saúde, considerando o interesse do estudo, em língua portuguesa.

Com isso, os critérios de inclusão para utilização dos textos e artigos analisados se basearam em credibilidade da fonte informativa e tema relacionado diretamente aos tópicos de interesse deste estudo. Assim, foram utilizados todos os artigos publicados sobre a temática aqui pesquisada e que estivessem em consonância com os questionamentos e objetivos traçados. Em contrapartida, os critérios de exclusão para construção do referencial teórico foram baseados nos artigos que não estavam relacionados com a temática, como também artigos com o ano de publicação abaixo de 2014, havendo necessidade de utilizar artigos a partir do ano de 2007, em virtude da ausência de publicações relacionados com a temática anterior a esse ano.
Vale considerar que, para dar maior aperfeiçoamento à revisão integrativa, foi realizado um refinamento. Esse processo buscou os aspectos que estão relacionados às palavras-chave: oncologia, neoplasia ovariana, técnica de preservação da fertilidade, reprodução assistida.

Nesse sentido, os dados encontrados foram tratados por meio de um quadro, a partir de agrupamento quantitativo de artigos, eixo de discussão, bases de dados, ano de publicação e referência. Com relação à discussão dos resultados, foi elaborado um quadro que aborda o detalhamento da pesquisa realizada na busca de artigos que tivessem total relação com o tema deste estudo, e os objetivos propostos para melhor identificar e conhecer o profissional de enfermagem, além das técnicas utilizadas na preservação da fertilidade, respectivamente.

\section{RESULTADOS}

Diante dos artigos analisados e utilizados, entende-se que a atuação do enfermeiro frente à preservação da fertilidade em pacientes com câncer de ovário é primordial, visto que é o enfermeiro que possui a maior acessibilidade ao paciente.

Para a construção da tabela de filtragem abaixo, seguiu-se os seguintes critérios para os artigos pesquisados:

- Critério de Filtragem 1: Números de artigos encontrados;

- Critério de Filtragem 2: Texto Completo, País (Brasil), Idioma (Português), Ano de publicação abaixo de 2016 e Tipo de trabalho (Artigo);

- Critério de Filtragem 3: Base de dados: LILACS, SCIELO;

- Critério de Filtragem 4: Artigos relacionados à temática do estudo;

- Critério de Filtragem 5: Artigos relacionados com o objetivo proposto. 


\begin{tabular}{|c|c|c|c|c|c|c|}
\hline Item & Palavras-Chave & Critério 1 & Critério 2 & Critério 3 & Critério 4 & Critério 5 \\
\hline 1 & Oncologia & 33.463 & 187 & 187 & 1 & 1 \\
\hline 2 & Neoplasias Ovarianas & 70.582 & 2 & 2 & 1 & 1 \\
\hline 3 & $\begin{array}{l}\text { Técnicas da preservação da } \\
\text { fertilidade }\end{array}$ & 1.287 & 5 & 5 & 2 & 1 \\
\hline \multirow[t]{2}{*}{4} & Reprodução Assistida & 9.031 & 39 & 39 & 1 & 1 \\
\hline & Total & 114.363 & 344 & 344 & 5 & 4 \\
\hline
\end{tabular}

Tabela 1 - Critérios de filtragem dos artigos pesquisados na BVS.

Fonte: Autoria própria (2018).

\begin{tabular}{|c|c|c|c|c|c|}
\hline $\begin{array}{c}\mathrm{N}^{\circ} \text { de artigos } \\
\text { utilizados }\end{array}$ & Questionamentos & $\begin{array}{l}\mathrm{N}^{\circ} \text { de artigos } \\
\text { relacionados com } \\
\text { a temática do } \\
\text { estudo }\end{array}$ & Ano & $\begin{array}{l}\text { Base de } \\
\text { dados }\end{array}$ & $\begin{array}{c}\text { Referências dos artigos } \\
\text { pesquisados }\end{array}$ \\
\hline 1 & $\begin{array}{l}\text { Orientação dos } \\
\text { profissionais } \\
\text { de saúde sobre } \\
\text { fertilidade }\end{array}$ & 00 & - & - & - \\
\hline 2 & $\begin{array}{l}\text { Orientações do } \\
\text { enfermeiro sobre } \\
\text { fertilidade }\end{array}$ & 01 & 2013 & LILACS & $\begin{array}{l}\text { FLÓRIA-SANTOS, Milena et } \\
\text { al. Atuação do enfermeiro em } \\
\text { oncologia na perspectiva da } \\
\text { genética e genômica. Texto \& } \\
\text { Contexto Enferm, v. 22, n. 2, p. } \\
\text { 526-533, 2013. }\end{array}$ \\
\hline
\end{tabular}

Quadro 1 - Critérios de filtragem dos artigos pesquisados na BVS.

Fonte: Autoria própria (2018) 


\begin{tabular}{|c|c|c|c|c|c|}
\hline $\begin{array}{c}\mathrm{N}^{\circ} \text { de artigos } \\
\text { utilizados }\end{array}$ & Objetivos & $\begin{array}{c}\mathrm{N}^{\circ} \text { de artigos } \\
\text { relacionados com } \\
\text { a temática do } \\
\text { estudo }\end{array}$ & Ano & $\begin{array}{l}\text { Base de } \\
\text { dados }\end{array}$ & $\begin{array}{c}\text { Referências dos artigos } \\
\text { pesquisados }\end{array}$ \\
\hline \multirow{4}{*}{04} & $\begin{array}{c}\text { Identificar } \\
\text { na literatura } \\
\text { nacional o } \\
\text { quantitativo de } \\
\text { publicações } \\
\text { sobre a atuação } \\
\text { do enfermeiro } \\
\text { diante do } \\
\text { diagnóstico de } \\
\text { câncer ovariano }\end{array}$ & 01 & 2013 & LILACS & $\begin{array}{c}\text { SANTIAGO, Diego } \\
\text { Lobão; LIMA, Mauricio } \\
\text { Batista; VENICIUS, Marcio. } \\
\text { Disgerminoma em gestação: } \\
\text { conduta conservadora. Rev. } \\
\text { Paraense, de Medicina, v. 27, n. } \\
\text { 3, } 2013 .\end{array}$ \\
\hline & \multirow{3}{*}{$\begin{array}{c}\text { Conhecer } \\
\text { a fonte de } \\
\text { informação que } \\
\text { esses pacientes } \\
\text { portadores de } \\
\text { câncer ovariano } \\
\text { tiveram acerca } \\
\text { das técnicas de } \\
\text { preservação }\end{array}$} & \multirow{3}{*}{03} & 2013 & LILACS & $\begin{array}{c}\text { MARINHO, Ricardo, Mello et } \\
\text { al. Preservação da fertilidade } \\
\text { em mulheres com câncer: } \\
\text { atualização e perspectivas. Rev. } \\
\text { Med Minas Gerais, p. 511-515, } \\
\text { 2013. }\end{array}$ \\
\hline & & & 2013 & LILACS & $\begin{array}{c}\text { SANTIAGO, Diego } \\
\text { Lobão; LIMA, Mauricio } \\
\text { Batista; VENICIUS, Marcio. } \\
\text { Disgerminoma em gestação: } \\
\text { conduta conservadora. Rev. } \\
\text { Paraense, de Medicina, v. 27, n. } \\
\text { 3, } 2013 .\end{array}$ \\
\hline & & & 2013 & $\begin{array}{l}\text { Rev. Saúde e } \\
\text { Pesquisa }\end{array}$ & $\begin{array}{l}\text { LUNARDI, Franciele Osmarini; } \\
\text { FERREIRA, Isabela Seabra; } \\
\text { BERNUCl, Marcelo Picinin. } \\
\text { Banco de Ovário: Uma } \\
\text { Alternativa para Preservação } \\
\text { de Fertilidade em Mulheres } \\
\text { Diagnosticadas com Câncer. } \\
\text { Rev. Saúde e Pesquisa, v. 6, n. } \\
\text { 3, } 2013 .\end{array}$ \\
\hline
\end{tabular}

Quadro 2 - Refinamento de artigos relacionados aos objetivos do estudo.

Fonte: Autoria própria (2018)

De acordo com o levantamento dos artigos descritos nos Quadros 1 e 2, foi observado que os assuntos abordados no trabalho estão presentes, auxiliando na construção para um melhor esclarecimento do tema. Para uma melhor compreensão desses quadros, ressalta-se que os artigos em destaque foram pesquisados na BVS e na biblioteca eletrônica Scielo. Os artigos que foram abordados neste estudo citam os assuntos pertinentes no campo da atualidade, visando assim um melhor entendimento de cada temática, considerando os fatos mais atuais em cada eixo.

Buscou-se, no entanto, levantar informativos e estudos sobre a atuação do enfermeiro diante do diagnóstico de câncer e acerca das técnicas de preservação da fertilidade. No entanto, houve certa dificuldade, devido à ausência de publicação relacionada ao tema.

Os artigos encontrados tratam do diagnóstico precoce da doença, quando se tem 
a maior possibilidade de garantir a fertilidade antes do início do tratamento da doença com radioterapia e quimioterapia. Além disso, apresentam algumas complicações para o paciente após o início do tratamento, e ainda abordam as técnicas de fertilidade de ambos sexos, associadas à reprodução assistida (SANTIAGO; LIMA; VENICIUS, 2013).

O uso das técnicas de criopreservação de embriões, de oócitos, de tecido ovariano, supressão da função ovariana e de transposição ovariana foram as técnicas encontradas nesses artigos para promover a preservação da fertilidade. Essas técnicas são utilizadas a fim de garantir a reprodução após o tratamento, tendo em vista que grande parte das pacientes que são acometidas por uma doença neoplásica irão sobreviver a ela e poderão manifestar desejo reprodutivo no futuro (FERREIRA; SOARES; MOTTA, 2011).

Para que ocorra a orientação ao paciente portador de neoplasias ovarianas, é fundamental que o profissional da área da saúde tenha conhecimento das técnicas de preservação da fertilidade. Essas técnicas podem ser encontradas nos artigos abordados neste trabalho, e o melhor méto do deverá ser estudado junto ao paciente, associando esse às técnicas de fertilização in vitro e posterior congelamento de embriões (SANTIAGO; LIMA; VENICIUS, 2013). Contudo, as técnicas são consideradas recentes e ainda apresentam resultados incertos em fases experimentais (MARINHO, 2013).

O trabalho desenvolvido neste estudo mostra que devemos refletir sobre a atuação dos profissionais de enfermagem frente aos desejos dos pacientes oncológicos, visto que eles têm maior acesso ao paciente. $O$ enfermeiro deve ser capaz de avaliar fatores protetores e preditivos, considerando os casos de origem genética, incluindo a história familiar e pessoal para que se possa obter informações de qualidade a fim de garantir a saúde, prevenção, rastreamento, diagnóstico, prognóstico, seleção de tratamento e monitoramento da eficácia do tratamento (FLORIA-SANTOS, 2013).

Dessa forma, o profissional que faz o atendimento ambulatorial ou primário precisa de uma formação elencada em várias abordagens de estudo sobre o paciente oncológico com neoplasia ovariana, pois, como parte da educação e consentimento informado antes da terapia do câncer, os profissionais de saúde da equipe multiprofissional devem abordar a possibilidade de infertilidade com pacientes tratados durante seus anos reprodutivos. Além de estar preparado para discutir as opções de preservação da fertilidade e/ou encaminhar todos os pacientes em potencial para os especialistas em reprodução (LOREN et al., 2013).

Portanto, a atuação do enfermeiro junto a equipe multiprofissional e interdisciplinar precisa considerar o cuidado, priorizando os recursos. E, com isso, integrar os seus conhecimentos na assistência no ensino e em pesquisas em oncologia.

\section{CONSIDERAÇÕES FINAIS}

Diante do estudo levantado, percebeu-se a relevância da atuação do enfermeiro no atendimento a pacientes oncológicos, tendo em vista a importância de um serviço de qualidade, originada de uma formação contínua. Todavia, sabe-se que os enfermeiros que buscam uma educação continuada almejam, além do conhecimento técnico complementar, engajar-se na prática não só individual, mas também coletiva. Além disso, buscam a reflexão sobre a própria prática, para pensar sobre "o saber fazer", o que resulta no refazer de suas ações para um atendimento qualificado na enfermagem.

Dessa forma, os profissionais de enfermagem necessitam, como em qualquer outra profissão, de uma educação continuada, em que se possa somar conhecimento e aprimorar técnicas para que possam atuar de forma efetiva. Ou seja, para poder contribuir positivamente com orientação, planejamento, 
direcionamento e encaminhamento, o enfermeiro precisa buscar conhecimento e se atualizar rotineiramente.

A atuação desse profissional é de extrema importância para o campo da oncologia, pois é ele que acolhe, orienta e proporciona expectativas para uma possível cura. Nesse contexto, ao analisarmos os artigos que abordam diagnóstico, tratamento desta patologia e apresentam as técnicas de preservação da fertilidade, percebemos que a orientação por parte dos enfermeiros sobre essas técnicas à pacientes com diagnóstico de câncer de ovário é deficiente.

No desenvolvimento deste estudo, houve dificuldade de encontrar artigos relacionados à temática, ou que respondessem aos nossos objetivos e questionamentos. E pouco foi citado nesses trabalhos sobre a orientação feita pelos enfermeiros ou qualquer outro profissional da saúde a respeito da questão da fertilidade.

Com isso, percebemos que há necessidade de publicações sobre a atuação do enfermeiro em relação ao direcionamento de pacientes frente às técnicas de preservação da fertilidade em mulheres com câncer de ovário. Da mesma forma, verificamos a necessidade de uma formação continuada e focada na oncologia. 


\section{REFERÊNCIAS BIBLIOGRÁFICAS}

CASTELLOTTI, D. S.; CAMBIAGHI, A. S. Preservação da fertilidade em pacientes com câncer. Rev. Bras. Hematol. Hemoter., v. 30, n. 5, p. 406-410, 2008.

FERREIRA, F. P.; SOARES, J. J. M.; MOTTA, E. L. A. da. Preservação da fertilidade: a importância de oferecer esta possibilidade às pacientes com doenças neoplásicas. Rev. Bras. Ginecol. Obstet., v. 33, n. 9, p. 223-226, 2011.

FLORIA-SANTOS, M. Práctica de lo enfermero en oncología en la perspectiva de la genética y genómica. Texto contexto - enferm., v. 22, n. 2, p.526-533, 2013.

HARTT, V. Técnicas ajudam pacientes a vencer a doença sem abandonar o sonho de ter filhos. Instituto vencer o câncer.

2014. Disponível em: <http://vencerocancer.com.br/pacientes/ qualidade-de-vida-2/efeitos-colaterais/tecnicas-ajudam-criancas-ejovens-pacientes-vencer-doenca-sem-abandonar-o-sonho-de-terfilhos >. Acesso em: 20 ago. 2018.

INSTITUTO NACIONAL DO CÂNCER - INCA. Tipos de câncer. Rio de Janeiro: INCA, [2018]. Disponível em:<http://www2.inca.gov.br/ wps/wcm/connect/tiposdecancer/site/home/ovario>. Acesso em: 20 ago. 2018.

. Estimativa da incidência e mortalidade por câncer no Brasil 2018-2019. Rio de Janeiro: INCA, 2018. Disponível em:< http://www. inca.gov.br/estimativa/2018/index.asp>. Acesso em: 20 ago. 2018

LOREN, A. W. L. et al. Fertility preservation for patients with cancer: American Society of Clinical Oncology clinical practice guideline update. J Clin Oncol., v. 31, n. 19, p. 2500-2510, jul. 2013. doi: 10.1200/JCO.2013.49.2678.

LUNARDI, F. O.; FERREIRA, I. S.; BERNUCI, M. P. Banco de Ovário: Uma Alternativa para Preservação de Fertilidade em Mulheres Diagnosticadas com Câncer. Rev. Saúde e Pesquisa, v. 6, n. 3, 2013.

MARINHO, R. M. Preservação da fertilidade em mulheres com câncer: atualização e perspectivas. Rev. Med Minas Gerais, p. 511 515,2013

MENDES, K. D. S.; SILVEIRA, R. C. C. P.; GALVAO, C. M. Revisão integrativa: método de pesquisa para a incorporação de evidências na saúde e na enfermagem. Texto contexto - enferm., Florianópolis, v. 17, n. 4, Dec. 2008. 
ROSA E SILVA, A. C. J. S. Preservação de fertilidade. Rev. Bras.

Ginecol. Obstet., v. 28, n. 6, p. 365-372, 2006.

SANTIAGO, D. L.; LIMA, M. B.; VENICIUS, M. Disgerminoma em gestação: conduta conservadora. Rev. Paraense, de Medicina, v. 27, n. 3 jul./set. 2013. 